\title{
A framework for adaptive opportunistic forwarding in wireless networks
}

\author{
Niki Gazoni ${ }^{+}$, Vangelis Angelakis ${ }^{+}$, Raffaele Bruno ${ }^{*}$, Marco Conti ${ }^{*}$, Vasilios Siris ${ }^{+}$ \\ ${ }^{+}$ICS-FORTH, N. Plastira 100, Vassilika Vouton, 70013 Heraklion, Greece \\ *IIT-CNR, Via G. Moruzzi 1-56124 Pisa, Italy \\ \{angelak,ngazoni,vsiris\}@ics.forth.gr, \{raffaele.bruno,marco.conti\}@iit.cnr.it
}

\begin{abstract}
We present our initial work on the design and of an opportunistic forwarding scheme for a wireless network. The main focus in our work is to take advantage of the interplay of the lower layer parameters and thus decide the design aspects of the forwarding decisions for an adaptive probabilistic opportunistic forwarding scheme. We have already shown that our opportunistic scheme can outperform single path routing for moderate channel error values, we have also identified a tradeoff between differentiating the forwarder's back-off value to avoid collisions and reducing delay.
\end{abstract}

\section{Introduction}

In opportunistic routing multiple paths from a source towards a destination are used to convey the data packets. These paths are used to simultaneously carry the same packet. In wireless networks where the medium is broadcast the notion of paths becomes loose. Transmitters typically broadcast their packets with sufficient control information so that receiver nodes can decide whether or not rebroadcasting the received packets will help in reaching the destination.

In the design of an opportunistic routing scheme, there are two key design decisions: (i) how the receiver of a packet will decide whether to forward or not and (ii) provided that a receiver decided to forward a packet, when is the most appropriate time to do so.

In ExOR [1] forwarding is decided after the packet has been received by a subset of nodes, according to a forwarding list that is generated by the current transmitter. Nodes need to discover and agree on which nodes received the packet, and only the node in that set that is "closest" to the destination forwards the packet. After collecting of a batch of packets, which is uniquely identified by a BatchID, the source broadcasts each packet in the batch, listing the forwarding nodes in priority order, in the packet's header. Due to the centralized coordination and scheduling that is needed between forwarders and the destination, ExOR introduces high overhead when the batch of packets to transmit is small as in bursty and short-lived flows, or the number of candidate forwarders is large.
SOAR [2] was proposed as an improvement to the ExOR protocol in order to support multiple flows. In SOAR the candidate forwarders are constrained to be along or near the shortest path from source to destination. A significant difference between ExOR and SOAR is that SOAR performs the routing decision process on a per-packet basis rather than on an entire batch. Finally, in SOAR the forwarder list is limited to 5 relays. Overall, SOAR incurs slightly less overhead than ExOR and restricts flows as close to the shortest path from source to destination as possible. To make the protocol reliable to ACK losses, selective ACKs are used. However, the increase in throughput that is observed when SOAR is in use is partly due to its opportunistic scheme and partly due to a complex acknowledgment scheme employed, making unclear to what extent this protocol contributes to opportunistic routing.

The Parametric probabilistic sensor network routing scheme of [3] proposes two protocols that forward a single packet, with varying retransmission probability, through a network of sensor nodes, focusing on simplicity and robustness to errors in distance estimation. A "Destination Attractor" assigns a higher retransmission probability to the packet as it moves closer to the destination and reduces it as the packet moves away from the destination.. The "Directed Transmission" scheme improves probabilistic routing's performance by assigning exponentially higher forwarding probabilities to nodes that are on the shortest path from the source to the destination and decreasing it as the packet strays from the shortest path. This leads to lower resource consumption than Destination Attractor and can be tuned to resemble shortest path routing, when the misinformation is low enough.

In [4], it is emphasized that the throughput gain achieved by opportunistic routing protocols is not clearly attributed to the opportunistic selection of next hops but is also partly due to elaborate acknowledgement or scheduling features that can well be implemented on traditional routing. Moreover, the authors point out the risk of duplicate forwarding that is experienced in opportunistic schemes, in cases when the multiple forwarders are unaware of others' transmissions, leading to collisions and potentially more retransmissions than single path routing. It is also demonstrated that use of ETX metric by an opportunistic scheme can result to low performance when node

978-1-4244-4439-7/09/\$25.00 C2009 IEEE 
coordination is imperfect. Finally, it is observed that in low network density there are no significant improvements by using opportunistic routing. The theme of this work is to identify points of failure in previously presented protocols.

Overall, previously suggested opportunistic protocols can be characterized by lack of concrete understanding of the way key wireless networking primitives and design decisions affect the performance of an opportunistic routing scheme. In order to study how these primitives affect an opportunistic routing scheme and to facilitate our own scheme design we have developed a framework in which using simulation together with mathematical analysis we will be provided with this required insight, while designing a new adaptive opportunistic forwarding scheme.

\section{System model}

The system model employed thus far in this study is that of a TDM based communication with the assumptions of

i. Fixed slot size

ii. Full topology knowledge by all nodes

iii. Local decision making -no central controllers exist

For simulation and analysis simplicity we have also made the simplifying assumption of a geometric propagation model: A transmission from a source is received by all nodes within a "communication radius", with a given reception probability for each potential receiver.

In this design a node key decisions are the forwarding probability assigned to a received packet and the back-off method employed.

The forwarding probability initially investigated is a linear one based on Euclidian distance (Figure 1) as a metric. Note that this can be replaced by any other path cost metric, as long as it has a known minimum and maximum, or such bounds can be tightly estimated.

Formally, this forwarding probability $p$ is expressed by:

$$
p=\frac{1}{d_{\text {min }}-d_{\text {max }}}\left[d \cdot\left(P_{\max }-P_{\min }\right)+P_{\text {min }} \cdot d_{\min }-P_{\max } \cdot d_{\max }\right]
$$

Where $d$ is the distance between the candidate forwarder and the destination, $P_{\max }$ is the forwarding probability associated to the nearest possible candidate (i.e., for $d=$ $\left.d_{\min }\right)$, and $P_{\min }$ is the probability associated to the furthest possible candidate (i.e., for $d=d_{\max }$ ). It is straightforward to observe that $0 \leq p \leq 1$ for $d_{\min } \leq d \leq d_{\max }$ if and only if $0 \leq$ $P_{\max } \leq 1$ and $0 \leq P_{\min } \leq 1$. Once a forwarding probability is assigned to a packet a random number of back-off timeslots is calculated with uniform probability in $\left[1, W_{i n} n_{\max }\right]$ where $W_{i n} n_{\max }$ is a maximum number of timeslots the packet may wait at a node before being transmitted. The $W_{i n}$ max value can either be a fixed number of maximum backoff slots, or may depend again on the probability assigned, with the rationale to help packets on "better" forwarding candidates to be transmitted earlier and thus reduce delays.

Initial simulations conducted with the pair $\left(P_{\max }=1\right.$, $\left.P_{\min }=0\right)$ indicated that a linear forwarding probability as the one expressed in formula (1) results in too few nodes having sufficiently high forwarding probability. As a consequence, we measured high delays.

To deal with this problem, we modified the initial forwarding probability function to increase the number of potential forwarding candidates having probability equal to one. This was achieved by using a piecewise function composed of flat region saturated to probability of one, and a linear region. Formally, this can be written as follows:

$$
p=\min \left\{1, \frac{1}{d_{\min }-d_{\max }}\left[d \cdot\left(P_{\max }-P_{\min }\right)+P_{\min } \cdot d_{\min }-P_{\max } \cdot d_{\max }\right]\right\}
$$

The piecewise function is produced by introducing $P_{\max }>1$, hence introducing more than one certain forwarders. Using simple algebraic manipulations, it is straightforward to derive from formula (2) that $p=1$ for $d^{\prime} \leq d \leq d_{\max }$, where

$$
d^{\prime}=\frac{d_{\text {min }}-d_{\text {max }}}{P_{\text {max }}-P_{\text {min }}} \cdot\left(P_{\text {max }} \cdot d_{\text {max }}-P_{\text {min }} \cdot d_{\text {min }}+1\right)
$$

Thus, adapting the $P_{\max }$ value we change the shape of the forwarding probability function. Intuitively, we may introduce more certain forwarders using a higher $P_{\max }$.

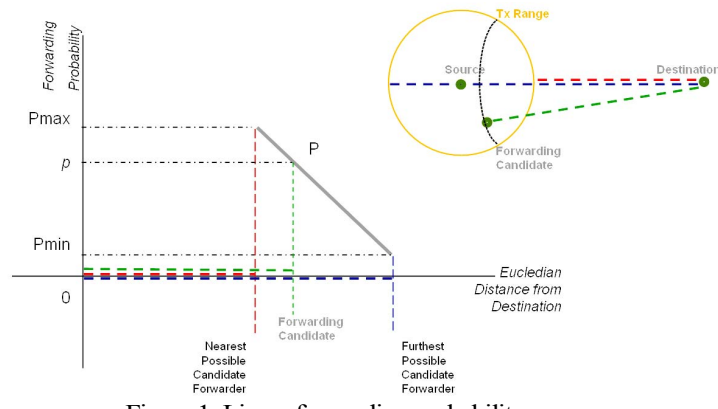

Figure 1: Linear forwarding probability.

Furthermore, we compared the performance of the linear probability function to that of a step-wise probability function in the presence of metric misestimation, which can occur due to the distributed nature of the scheme. In the step-wise probability function, nodes are either assigned a forwarding probability equal to 1 or a probability equal to zero, that is they make a hard decision on packet forwarding.

\section{Initial Results}

The forwarding probability function slope defines how great the difference in the probability to forward the packet will be between the neighbors of the node currently holding the packet. Specifically, the steeper the slope is, the neighbors closer to the destination will be given higher probability values. To ensure the packet's progressing to the destination, at least one neighbor with forwarding probability equal to 1 is needed; ideally this would be the neighbor on the shortest path to destination. As the PER increases, more certain forwarders are required, to make up for failed packet receptions, but the need arises to relax potential collisions 
among them by differentiating their back-off timers. Furthermore, for a given PER value, having more than one certain forwarders yields lower delay (see fig.2).

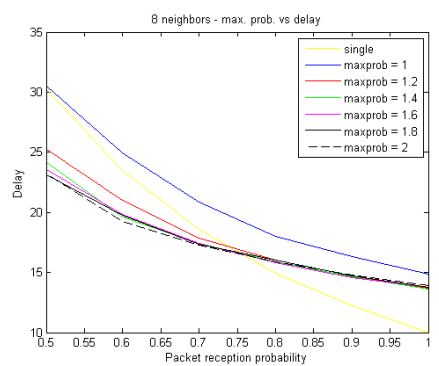

Figure 2: Effect of the slope of the forwarding probability -using a piecewise probability function with more than 1 certain forwarders.

Using a step-wise function consumes fewer resources than the linear function and it is also less affected by metric miscalculation. This is justified by the fact that even when inaccurate estimations are made few (if any) nodes will miscalculate their metric and fall below or go above threshold, whereas the majority of nodes will get the same probability as that they would get if there was no miscalculation. To further understand the impact miscalculations have on our scheme, we measured the delay over the same 10 hop path for both the linear and the step-wise probability function, for various packet error rate values. Results (fig. 3) demonstrate that metric miscalculation, when expressed as noise to the actual metric value, does not affect delay performance significantly for neither the linear nor the stepwise probability function. Metric noise was modeled as a randomly selected value from a uniform distribution, with a mean equal to the real value of the metric. The width of the metric values interval is equal to a percentage of the real value of the metric.

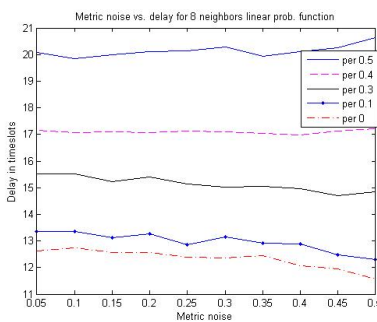

(a)

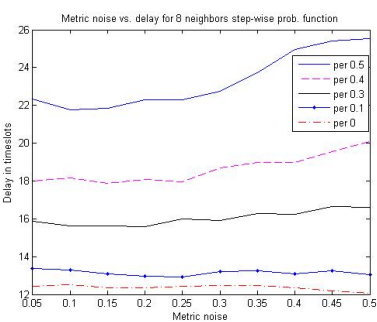

(b)
Figure 3: Metric noise has practically no affect on the decision, whether the forwarding function is (a) linear, or (b) step.

We employed two approaches to back-off window schemes. Using a fixed back-off window, all neighbors would randomly select their back-off values from the same range of idle slots. Differentiating, each node randomly selects its back-off values from a different range of idle slots. Specifically, the back-off window of a node will be randomly chosen from a set of fewer idle slots, the larger its forwarding probability is. There is a steeper increase in delay for the fixed back-off window scheme as the width of the back-off window interval increases, which renders fixed back-off values larger than 2 inefficient. It can be observed that the lowest delay is measured for a back-off window of 1 , at the cost of many unnecessary transmissions. As we further increase the range of the back-off window the flooding is limited to an area around the shortest path, while the delay slightly increases.

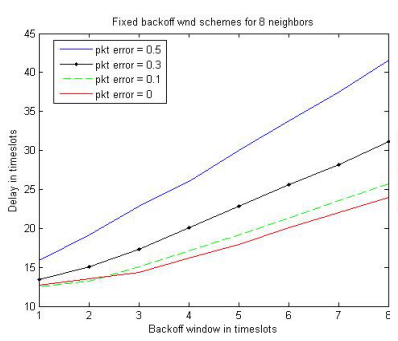

(a)

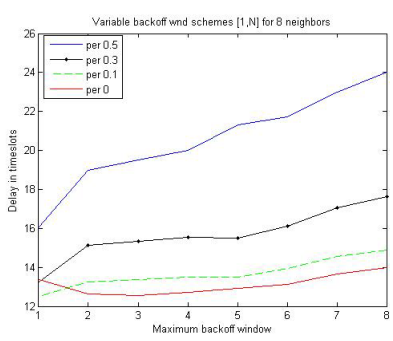

(b)
Figure 4: Backoff effect on Delay: (a) Fixed, (b) Variable [1..N] scheme

\section{Early conclusions}

We have already shown that our opportunistic scheme can outperform single path routing for error values larger than $20 \%-25 \%$ (fig. 2), for the optimized slope of the forwarding probability function, with a restrained use of resources. Furthermore, we have shown that the optimal manner of adapting to increasing error is to increase the number of forwarders by increasing the slope of the forwarding probability function. In particular, the number of certain forwarders has the most impact on performance and they need to be increased with respect to error conditions.

To reduce resource consumption -in terms of packet transmissions, utilizing a step-wise function is a sound approach, which proved robust to metric miscalculations. Finally, there is a tradeoff between differentiating each forwarder's back-off value to reduce resource consumption and reducing delay. Our results show that a variable backoff scheme that gives priority (smaller back-off windows) to best forwarders according to their forwarding probability is preferable to a fixed back-off window for all forwarders.

\section{References}

[1] S. Biswas and R. Morris, "Exor: opportunistic multi-hop routing for wireless networks," in SIGCOMM '05.

[2] E. Rozner, J. Seshadri, Y. Mehta, and L. Qiu, "Simple Opportunistic. Routing Protocol for Wireless Mesh Networks" in Proc. WiMesh'06.

[3] C. L. Barrett, S. J. Eidenbenz, L. Kroc, M. Marathe, and J. P. Smith. "Parametric Probabilistic Sensor Network Routing", in Proc. of ACM'07.

[4] Zhong, Z. and Nelakuditi, S., "On the efficacy of opportunistic routing." in: Proc. of 4th IEEE International Conference on Sensor and Ad Hoc Communications \& Networks, SECON '07. 